\title{
Influência de alimentos líquidos e sólidos no controle do apetite ${ }^{1}$
}

\author{
Influence of liquid and solid \\ foods on appetite control
}

Denise Machado MOURÃO2

Josefina BRESSAN²

\section{R E S U M O}

Este trabalho discute o papel dos alimentos líquidos e sólidos na ingestão alimentar, fundamentando-se em uma revisão crítica sobre o assunto. Utilizaram-se os principais bancos de dados nacionais e internacionais em saúde, entre eles, Medline/PubMed, Web of Science, Lilacs, SciELO. A busca bibliográfica compreendeu o período de 1980 a 2008. Os resultados atuais das pesquisas indicam que o estado físico do alimento pode influenciar o consumo alimentar, tanto a curto quanto a longo prazo, e que os alimentos líquidos exercem um menor poder sacietógeno, em comparação aos sólidos. Os possíveis mecanismos envolvidos nesse fraco controle do apetite pelos líquidos são: falta de mastigação, fase cefálica da ingestão menos pronunciada, esvaziamento gástrico mais rápido e fatores cognitivos. Conclui-se que o uso de alimentos líquidos, em especial bebidas energéticas, deve ser moderado tanto na prevenção como no tratamento da obesidade.

Termos de indexação: Apetite. Fome. Obesidade. Resposta de saciedade. Viscosidade.

This review discussed the influence of solid and liquid foods on food intake, based on a critical review of the subject. The main national and international health databases, Medline/PubMed, Web of Science, Lilacs and SciELO, were searched. Publications from 1980 to 2008 were included. The findings showed that the physical nature of the food can influence food intake, both in the short term and long term, with liquids being less satiating than solids. The possible mechanisms involved on this impaired satiety response to liquids are: absence of mastication, short cephalic phase, faster gastric emptying and cognitive factors. In conclusion, liquid foods, especially calorie-containing beverages, should be consumed in moderation in order to prevent and treat obesity.

Indexing terms: Appetite. Hunger. Obesity. Satiety response. Viscosity.

\footnotetext{
1 Artigo elaborado a partir da tese de D.M. MOURÃO, intitulada "Influência de alimentos líquidos e sólidos na saciação e na saciedade". Viçosa; 2006.

2 Universidade Federal de Viçosa, Departamento de Nutrição e Saúde. Av. PH Rolfs, s/n., 36570-000, Viçosa, MG, Brasil Correspondência para/Correspondence to: D.M. MOURÃO.E-mail:<dmmourao@gmail.com>.
} 


\section{N T R O D U Ç Ã O}

Diante da crescente incidência da obesidade na população mundial, muitas pesquisas vêm sendo desenvolvidas, no sentido de identificar os elementos que mais contribuem para esse fato. Dentre esses elementos, um fator significativo tem sido a relação entre o estado físico em que o alimento é ingerido e o ganho de peso corporal. Isso porque os alimentos podem ser classificados segundo suas diferenças sensoriais, propriedades físicas e químicas, as quais contribuem para a regulação do comportamento alimentar e, também, para a regulação do metabolismo energético ${ }^{1,2}$.

Segundo a Organização Mundial de Saúde, os alimentos líquidos levam a um maior risco para o ganho de peso corporal ${ }^{3}$. Também outros autores observaram que a ingestão de alimentos sólidos parece suprimir o apetite, por um período de tempo maior do que líquidos. Entretanto, o assunto é ainda muito controverso, visto que outros pesquisadores encontraram uma associação inversa ${ }^{4-6}$. Além disso, constatou-se que uma grande parte dos resultados das pesquisas não é conclusiva, e que os possíveis mecanismos envolvidos nesse fenômeno ainda não foram esclarecidos.

Assim, este artigo avaliou e discutiu, de forma sistemática, a associação entre o estado físico dos alimentos e a obesidade, no intuito de verificar se a hipótese de que os líquidos exercem um menor poder sacietógeno nos indivíduos possui suficiente embasamento científico. Para isso, foi realizada uma revisão das principais pesquisas sobre o assunto levantando-se seus pontos mais significativos. Na busca bibliográfica, que compreendeu o período de 1980 a 2008, foram utilizados os principais bancos de dados nacionais e internacionais em saúde; entre eles, Medline/ PubMed, Web of Science, Lilacs e SciELO. O tema foi desenvolvido de acordo com os seguintes tópicos: 1) obesidade e ingestão de líquidos; 2) fatores que podem influenciar a compensação energética no controle da ingestão alimentar (fatores fisioló- gicos e mecanismos corporais; propriedades físico-químicas dos alimentos; e fatores psicológicos, cognitivos e sociais); 3) possíveis mecanismos envolvidos nessa hipótese e; 4) comparação entre os efeitos dos alimentos líquidos e alimentos sólidos na saciedade.

\section{Obesidade e ingestão de líquidos}

A tendência de que um alto consumo de bebidas seja uma importante preocupação de saúde pública justifica-se pela hipótese segundo a qual a energia contida nas bebidas favoreceria um consumo energético maior, em relação aos alimentos sólidos ${ }^{3}$.

$O$ alto consumo de bebidas, especialmente refrigerantes, tem sido apontado por pesquisadores como um dos possíveis fatores que leva ao ganho de peso em vários países ${ }^{7-9}$. Desde 1978, a ingestão de refrigerantes tem aumentado em cerca de 40\% nos Estados Unidos da América (EUA). Dados do Departamento de Agricultura e Economia dos EUA indicam que mais de um terço do consumo de açúcar desta população é proveniente de líquidos ${ }^{10}$. Os refrigerantes carbonatados têm contribuído com cerca de 33,2\% deste aumento no consumo de açúcar ingerido diariamente na dieta de americanos maiores de 2 anos de idade, ao passo que sucos de frutas contribuíram com uma parcela adicional de 9,8\%. Em 1997, nos EUA, o consumo de refrigerantes foi estimado em cerca de 33 litros per capita, sendo apenas 24,0\% deste valor referente ao grupo de dietéticos ${ }^{8,10}$.

Na região de Piracicaba (SP) verificou-se que o consumo médio diário alcançou, aproximadamente, $230 \mathrm{~mL}$ de refrigerante e $550 \mathrm{~mL}$ de bebidas com adição de açúcar entre adolescentes da rede pública de ensino ${ }^{7}$.

Baseados nesses dados, vários autores acreditam que a adição de calorias à dieta, proveniente dos líquidos, possa aumentar a ingestão energética total. Isto foi demonstrado para café, bebidas alcoólicas, refrigerantes, sucos de fruta e 
leite ${ }^{8,11}$. Adicionalmente, verificou-se que a ingestão de alimentos em diferentes estados físicos provocou uma maior redução no consumo após a ingestão de alimentos sólidos, seguida de alimentos pastosos e líquidos, respectivamente ${ }^{12}$.

\section{Fatores que podem influenciar a compensação energética no controle da ingestão alimentar}

Os principais fatores fisiológicos e os mecanismos corporais relacionados à compen- sação energética são: 1) idade e sexo, relacionados aos processos de anorexia e às desordens alimentares, respectivamente; 2) peso corporal, influenciado pela fome, pela saciedade, pela preferência por determinados alimentos e pelos padrões de alimentação; 3) distensão e esvaziamento gástrico, intimamente relacionados aos processos de saciação e saciedade; 4) hormônios periféricos, relacionados aos processos de esvaziamento gástrico e ao transporte intestinal de açúcar, como a colecistocinina (CCK), peptídeo semelhante ao glucagon (GLP-1), e a insulina; 5)
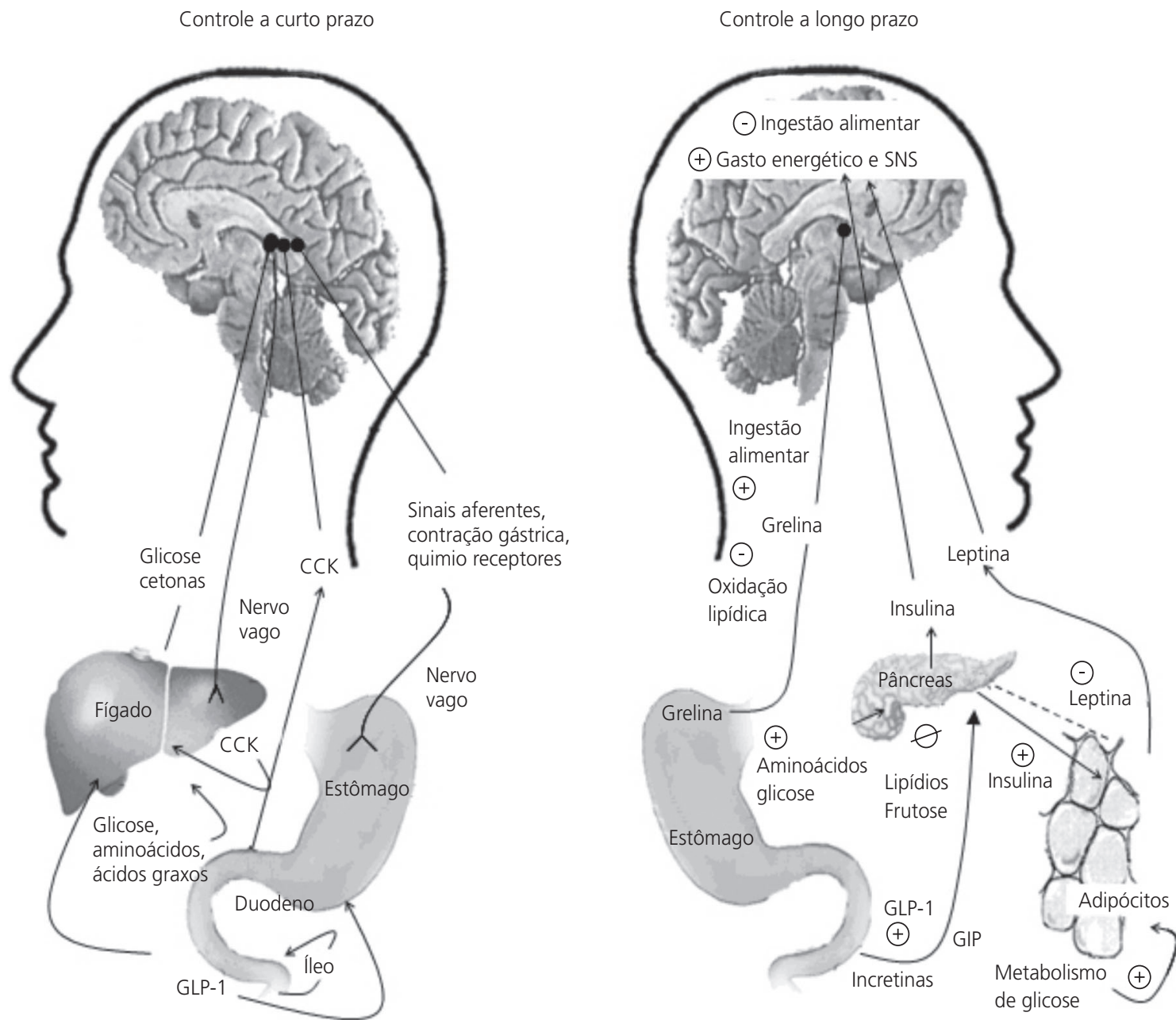

Figura 1. Principais fatores fisiológicos e mecanismos corporais relacionados ao controle da ingestão alimentar e à compensação energética, a curto e longo prazo.

Fonte: Adaptado de Havel${ }^{51}$.

Nota: seta contínua e/ou sinal positivo indicam estimulação, seta descontínua e/ou sinal negativo indicam inibição; CCK: colecistocinina; GLP-1: peptídeo semelhante ao glucagon; NPY: neuropeptídeo Y; GIP: peptídeo insulinotrópico glicose dependente; SNS: sistema nervoso simpático. 
hormônios e peptídeos centrais, também relacionados aos processos de esvaziamento gástrico, ao trânsito intestinal, à distensão gástrica e intestinal, ao nível de glicose sangüínea, e ao metabolismo hepático, influenciados pelo neuropeptídeo Y (NPY), leptina, galanina e grelina; 6) processos metabólicos, relacionados às taxas de oxidação de glicose e lipídios ${ }^{13}$. A Figura 1 mostra, esquematicamente, os principais fatores fisiológicos e os mecanismos corporais relacionados ao controle da ingestão alimentar e à compensação energética, a curto e a longo prazo.

Dentre as propriedades físico-químicas dos alimentos, a viscosidade, definida como a resistência que um fluido oferece ao movimento relativo de quaisquer de suas partes, também está relacionada a alterações na ingestão alimentar. Uma maior viscosidade retardaria o esvaziamento gástrico, aumentando o tempo de saciedade ${ }^{14}$. Portanto, de uma forma geral, alimentos mais viscosos tendem a retardar a sensação de fome por mais tempo do que alimentos menos viscosos.

A composição de macronutrientes também tem sido demonstrada como um fator que influencia a ingestão alimentar, na qual a proteína é o macronutriente mais sacietógeno, seguido de carboidratos e lipídios ${ }^{15}$, e a eficiência de absorção dos macronutrientes pode diferir, a depender do estado físico do alimento. Sólidos e líquidos apresentam diferenças em sua resistência à ação da degradação enzimática, bacteriana e mecânica, podendo os alimentos sólidos apresentar um menor aproveitamento energético. Além disso, um alto conteúdo de fibras, característica de alimentos sólidos, pode resultar também em perda de energia na absorção ${ }^{1}$. De forma semelhante, a saciedade pode ser afetada ainda pelo volume ${ }^{16}$ e pela densidade energética ${ }^{17}$, os quais interagem diretamente nos receptores gástricos e intestinais ${ }^{2}$.

Fatores psicológicos, como o condicionamento de comportamento restritivo diante de alimentos, podem surgir em indivíduos que fazem dieta constantemente, alterando, dessa forma, a ingestão alimentar. Fatores cognitivos, como o aprendizado da mastigação prolongada em determinadas culturas, parecem promover a saciação mais rapidamente. Socialmente, a acessibilidade a alimentos altamente energéticos, como os fast foods, e o aumento das porções ${ }^{18}$, dentre outros, também alteram a ingestão alimentar, contribuin-

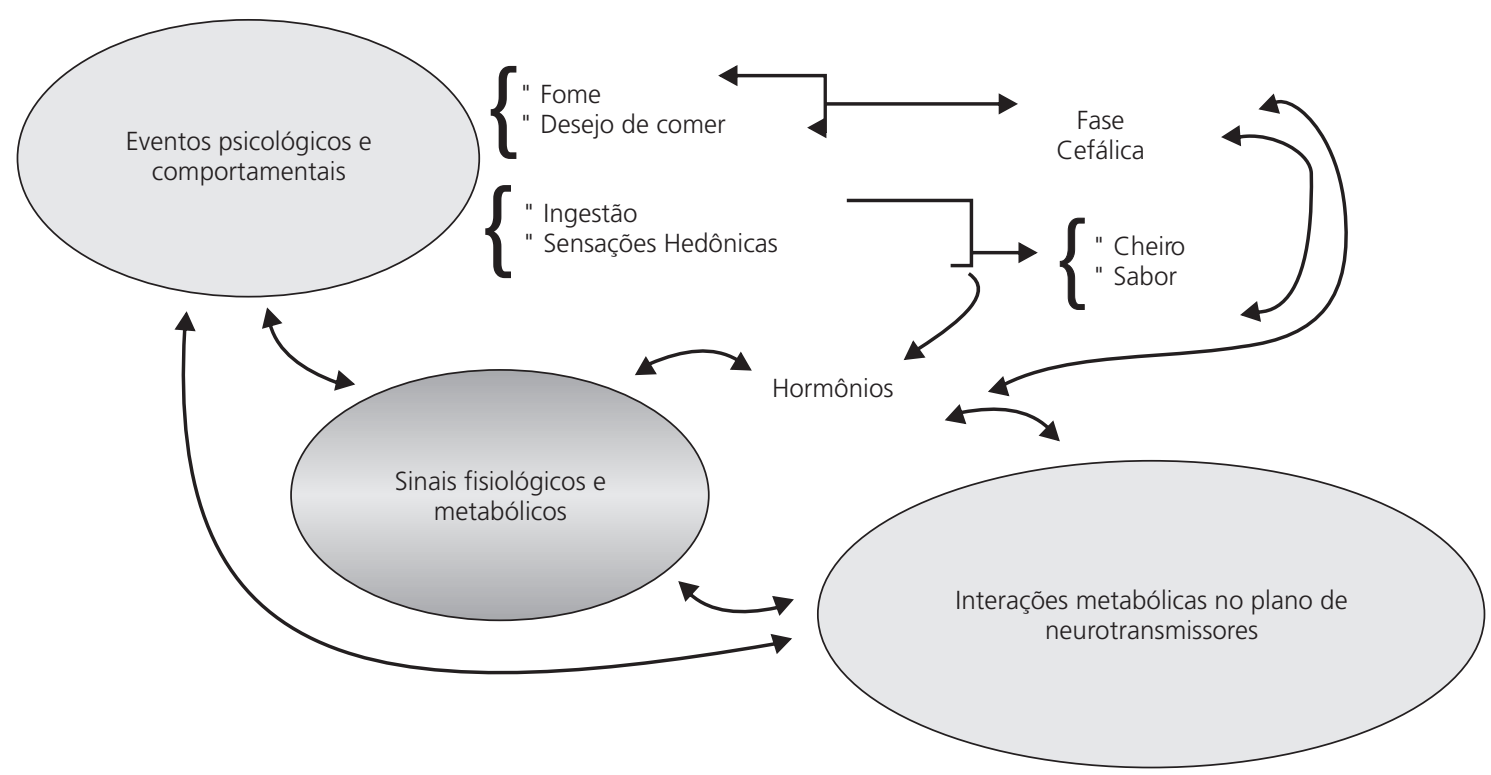

Figura 2. Rede de interações de um sistema psíquico-fisiológico. 
do para o ganho de peso ${ }^{19}$. A Figura 2 mostra esquematicamente uma rede de interações entre fatores psíquico-fisiológicos que controlam a ingestão alimentar.

\section{Possíveis mecanismos envolvidos na hipótese de que os alimentos líquidos saciam menos que os sólidos}

A ausência da mastigação, que ocorre quando da ingestão de alimentos líquidos, tem sido apontada como um dos fatores que contribuiriam para a menor saciedade. O tempo de exposição aos receptores orofaríngeos, intimamente ligados ao controle dos centros da fome a da saciedade, é muito maior para os alimentos sólidos do que para os líquidos ${ }^{20}$. Em ratos, verificou-se que o ato mecânico da mastigação promove a saciedade, especialmente em animais magros, quando comparados aos obesos ${ }^{21}$. Posteriormente, verificou-se, também em animais, que a mastigação ativa a liberação de histamina, a qual suprime fisiologicamente a ingestão alimentar, pela ativação dos centros de saciedade no hipotálamo. Com isso, há uma redução tanto do volume, quanto da velocidade de ingestão do alimento, um aumento da lipólise, particularmente em adipócitos viscerais e, ainda, um aumento da expressão gênica das proteínas desacopladoras (UCPs) ${ }^{22}$.

Esse menor tempo de exposição aos receptores orofaríngeos, que ocorre com alimentos líquidos, também resulta em uma fase cefálica da alimentação menos pronunciada, pois há uma fraca produção/liberação dos hormônios e peptídeos envolvidos. A palatabilidade, avaliação hedônica das propriedades sensoriais de um alimento ${ }^{23}$, tem sido considerada um importante e determinante fator na seleção e ingestão de alimentos em humanos ${ }^{24}$. Vários trabalhos mostraram que alimentos não palatáveis são menos ingeridos, quando comparados aos palatáveis ${ }^{23-25}$. Este último estudo sugeriu que pessoas com sobrepeso são mais susceptíveis à escolha de alimentos palatáveis, os quais, geralmente, têm mais energia, levando, possivelmente, a um aumento do peso corporal. Adicionalmente, foi verificado, também em humanos, que dietas não palatáveis promovem um estímulo reduzido do Sistema Nervoso Simpático (SNS), especialmente na fase inicial (cefálica) do processo de alimentação, podendo assim haver uma resposta diferenciada na termogênese ${ }^{26}$.

Quanto ao gasto energético, foi observada, após a ingestão de alimento sólido, uma maior resposta no metabolismo de repouso, quando comparada à ingestão do alimento líquido. Contudo, a dieta sólida utilizada foi mais aceita que a fórmula líquida ${ }^{27}$, devendo esses resultados ser interpretados com cautela. Posteriormente, Habas \& MacDonald ${ }^{28}$ encontraram um maior gasto energético, e níveis plasmáticos mais elevados de glicose e insulina após o consumo da dieta sólida, testada em relação à liquida. Entretanto, a dieta líquida desse estudo era hipertônica, o que, muito provavelmente, poderia ter levado a um retardo no esvaziamento gástrico da mesma. A falta de controle dessas duas variáveis nos trabalhos citados pode ter interferido na análise dos resultados, prejudicando assim a interpretação do efeito principal.

Um dos obstáculos no estudo é o fato de este tema estar relacionado a uma grande variedade de fatores, dificultando o controle dos mesmos. Testar uma mesma formulação alimentícia ou uma composição de alimentos nas formas líquida e sólida, de maneira que não haja diferenças significativas na densidade energética, na distribuição e no perfil de macronutrientes, na osmolalidade, no volume, entre outros, é uma tarefa árdua.

Ainda são limitadas as informações que possibilitariam esclarecer melhor o efeito da consistência dos alimentos na taxa de esvaziamento gástrico e na secreção de hormônios gastrointestinais. Já é conhecido que o esvaziamento gástrico de líquidos é mais rápido do que o de sólidos ${ }^{29}$. Entretanto, em refeições contendo quantidades normais de componentes sólidos e líquidos, há uma produção de solução viscosa, na qual as 
partículas sólidas ficam suspensas, à medida que esses componentes são misturados na boca e no estômago. Um aumento da viscosidade do conteúdo gástrico reduz a sedimentação dos sólidos no líquido e dificulta, assim, a habilidade preferencial que o antro tem em se esvaziar mais rapidamente de líquidos do que de sólidos ${ }^{30}$. Além disso, independentemente do tipo de alimento consumido, uma correlação negativa foi observada entre a taxa de esvaziamento gástrico e a saciedade ${ }^{31}$.

Deveria ser considerado, ainda, que mecanismos neurais, assim como a presença de produtos da digestão no duodeno, especialmente gordura e aminoácidos que estimulam a secreção de vários hormônios gastrointestinais, como a Colecistocinina (CCK), têm demonstrado ter um importante papel na regulação da ingestão alimentar. Reconhecido como o primeiro peptídeo anorexígeno, a CCK atua mais pronunciadamente na saciação do que na saciedade, assim como na ação de líquidos, comparado à ação dos alimentos sólidos ${ }^{32}$.

Alguns autores verificaram também que a concentração plasmática de noradrenalina apresentou tendência a ficar mais elevada após a ingestão de alimento sólido, em comparação à de líquidos, enquanto que a concentração de adrenalina, ao contrário, foi reduzida ${ }^{28}$. Alguns estudos consideram que a concentração plasmática de adrenalina, e não a de noradrenalina é reduzida em indivíduos obesos, tanto em repouso quanto após estimulação ${ }^{33}$. Entretanto, estudos com pré-obesos e pós-obesos indicaram que uma diminuição na estimulação da secreção de adrenalina, em pós-obesos, não está associada ao estado de obesidade, mas talvez já esteja presente em indivíduos pré-obesos ${ }^{34}$.

A leptina também tem demonstrado possuir forte influência na ingestão alimentar. Ela promove o decréscimo do consumo, pela sinalização da saciedade no cérebro. Em humanos, a leptina está associada ao peso corporal, ao índice de massa corporal e ao percentual de gordura corporal, sendo que uma falha na produção de leptina ou uma resistência à sua ação pode resultar em aumento de peso corporal ${ }^{35}$. Os níveis de leptina parecem ser maiores com o excesso de peso crônico, e reduzidos em jejuns prolongados ou restrições energéticas. Dietas ricas em lipídios proporcionam um aumento significativo na concentração sérica de leptina, porém isso não ocorre com o aumento da energia total ingerida, ou com dietas hiperprotéicas ${ }^{36}$.

A grelina, um peptídeo gastrintestinal, é outro exemplo de um importante hormônio relacionado à regulação, tanto do metabolismo energético, quanto da ingestão alimentar. Ao contrário da leptina, a grelina promove o aumento do consumo $^{37}$. Em estudos nos quais a grelina foi administrada em ratos, observou-se um aumento do quociente respiratório, sugerindo uma mudança do padrão metabólico em direção à glicólise, ao invés da oxidação de ácidos graxos, favorecendo assim a adiposidade e o ganho de peso ${ }^{38}$. Outros efeitos da grelina foram demonstrados, como estimulação da motilidade gástrica e secreção ácida ${ }^{39}$, estímulo do apetite ${ }^{40}$, entre outros. Em humanos, os níveis plasmáticos de grelina parecem estar aumentados no jejum ${ }^{38,41}$ e, ao contrário do que se esperava, reduzidos em obesos ${ }^{42}$. Uma possível explicação para este decréscimo na concentração plasmática de grelina em obesos seria pela ocorrência de uma adaptação fisiológica ao balanço energético positivo, associada à obesidade $^{40,42}$. Tem sido proposto, ainda, que a grelina é um hormônio que contribui para a iniciação de uma refeição ${ }^{41}$, uma vez que seus níveis plasmáticos parecem estar aumentados antes de uma refeição e diminuídos logo após a ingestão ${ }^{42}$. Uma maior e mais prolongada redução dos níveis de grelina foi verificada em idosos que receberam $25 \%$ de suas necessidades energéticas na forma de barras energéticas, alimento sólido, quando comparada a shakes, alimento liquido testado ${ }^{43}$. Entretanto, ainda não foram realizados trabalhos específicos, com delineamento adequado, para uma investigação dos níveis desses hormônios relacionados à ingestão de alimentos sólidos e líquidos e às suas influências, a curto e longo prazo, na fome. 
Influências cognitivas também devem ser consideradas como um fator importante na regulação da ingestão alimentar ${ }^{13,29}$. As áreas do cérebro nas quais o prazer ou os valores afetivos relacionados ao cheiro e ao gosto, estão intimamente relacionadas às áreas que envolvem as emoções. Na natureza, a energia normalmente não é originada de alimentos líquidos, com exceção do leite materno na infância. Nesta fase, ocorre uma grande e direta relação entre a viscosidade dos alimentos e a quantidade de energia. Dessa forma, o leite materno talvez promova uma importante fase de indução de um comportamento reflexo de que substâncias mais espessas contenham mais energia ${ }^{20}$. Ao longo dos anos, se incorpora que, ao consumir um líquido, se está satisfazendo a sensação de sede, e não a de fome. Adicionalmente, o fato de que a ingestão de um líquido, geralmente, ocorre junto com uma refeição, ou em intervalos ao longo do dia, e não em substituição a uma refeição principal, também contribui para essa associação cognitiva. Todas essas considerações podem significar que a diferença na resposta de saciedade, entre alimentos líquidos e sólidos, é baseada, entre outros fatores, em um comportamento adquirido 20,44 . A Figura 3 esquematiza os possíveis mecanismos envolvidos na interferência de alimentos líquidos na fome.

\section{Alimentos líquidos $\mathrm{x}$ alimentos sólidos na saciedade}

Alguns estudos têm demonstrado que líquidos promovem maior saciedade que sóli$\operatorname{dos}^{4-6,44}$. Entretanto, na grande maioria dos trabalhos que verificaram esse resultado foram utilizadas sopa ou formulações tipo shake, a serem consumidas como uma pré-carga, anteriormente a uma refeição, ou como a própria refeição teste.

As sopas são consideradas uma categoria de alimentos bastante heterogênea, apresentando propriedades completamente diferentes de bebidas ${ }^{12}$. Em geral, elas diferem das bebidas na composição nutricional, na temperatura e na forma de apresentação a ser consumida. Elas também
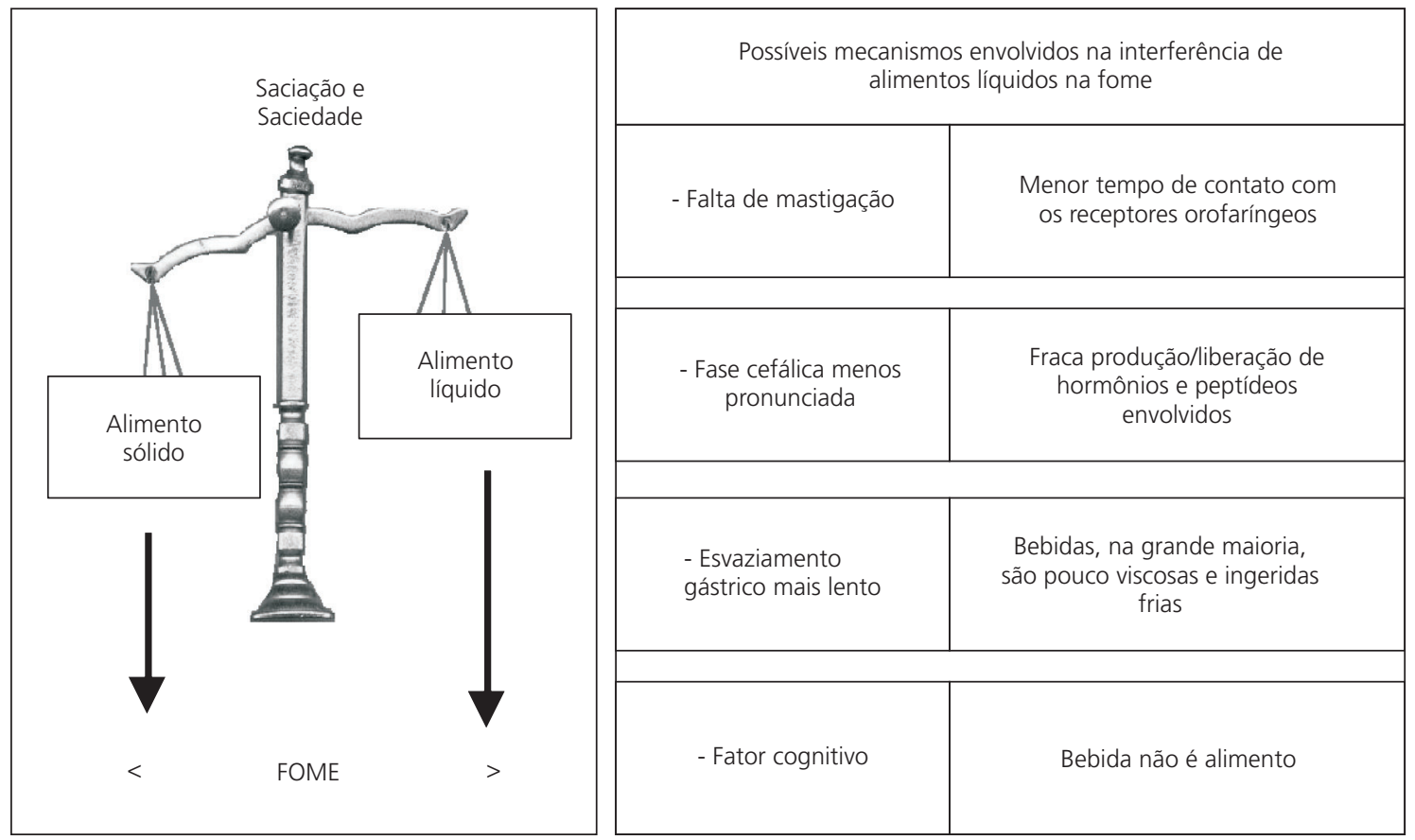

Figura 3. Possíveis mecanismos envolvidos na interferência de alimentos líquidos na fome. 
são consideradas, cognitivamente, como uma refeição. Dessa maneira, os estudos que utilizaram a sopa como dieta líquida, a ser contrastada com sólidos, tiveram um delineamento experimental completamente diferente daqueles com bebidas, não devendo assim ser comparados ${ }^{2,29}$.

Estudos com iogurte ${ }^{45}$ e formulações tipo shake 20,46,47 também não são adequados para comparar a influência de alimentos sólidos e líquidos na saciedade, mas sim a interferência de alimentos mais ou menos viscosos, na saciedade, visto que são caracterizados pela adição de agentes emulsificantes em uma das dietas a serem testadas. Adicionalmente, verificou-se que é comum na literatura a falta de controle de outros fatores importantes no controle da saciedade, como: composição de macronutrientes, peso, volume, temperatura, palatabilidade, conteúdo e estrutura de fibras, impressão cognitiva, entre outros, os quais podem impossibilitar interpretações conclu$\operatorname{sivas}^{1,2,29}$.

Por outro lado, outros estudos ${ }^{48,49}$ que utilizaram alimentos sólidos versus alimentos líquidos em seu delineamento, verificaram uma maior saciedade após a ingestão de sólidos. Também foi verificado que, quando a maior parte das calorias ingeridas era na forma de líquidos, a compensação energética não era observada, ao passo que com sólidos, os indivíduos restringiam seu consumo posterior ${ }^{49}$. Outros dois estudos De Graaf \& Hulshof ${ }^{45}$ e Hulshof et al. ${ }^{50}$ também verificaram uma maior saciedade após a ingestão de alimentos sólidos, comparada à de líquidos. Entretanto a utilização de agente emulsificante e de artefatos,

Quadro 1.Principais estudos comparativos, de 1980 a 2008, entre estado físico de alimentos e seus efeitos na ingestão alimentar.

\begin{tabular}{|c|c|c|c|c|}
\hline Alimentos comparados & Resultados elementos com potencias efeitos nos resultados* & Sexo & $\mathrm{n}$ & Referência \\
\hline${ }^{* *}$ Sopa $x$ vegetais & $\begin{array}{l}\text { Sopa promoveu maior saciedade/Diferença na temperatura dos } \\
\text { alimentos servidos }\end{array}$ & M & 22 & França ${ }^{4}$ \\
\hline Sopa $x$ vegetais cozidos com queijo & $\begin{array}{l}\text { Sopa promoveu maior saciedade/Diferença na temperatura dos } \\
\text { alimentos servidos }\end{array}$ & M & 8 & Itália ${ }^{6}$ \\
\hline Sopa x Risoto & $\begin{array}{l}\text { Sopa reduziu a fomeNariação do volume, densidade energética, } \\
\text { aparência e forma das dietas }\end{array}$ & $\mathrm{F}$ & 24 & EUA $^{5}$ \\
\hline Refrigerante $\mathrm{x}$ doce & $\begin{array}{l}\text { Redução no consumo após ingestão do alimento sólido/Va- } \\
\text { riação do tempo de ingestão e impacto do doce na sede }\end{array}$ & $\mathrm{F}$ & $\begin{array}{l}8 \\
7\end{array}$ & $\mathrm{EUA}^{49}$ \\
\hline Shakes mais ou menos viscosos & $\begin{array}{l}\text { Maior redução da fome após shake mais viscosos/Faixa etária } \\
\text { muito ampla (48 variações de13 anos) }\end{array}$ & $\mathrm{F}$ & $\begin{array}{l}54 \\
30\end{array}$ & $\mathrm{EUA}^{46}$ \\
\hline Fruta $x$ sopa $\times$ suco & $\begin{array}{l}\text { Saciedade decrescente: Fruta > sopa > suco/Diferenças na tem- } \\
\text { peratura e no volume dos alimentos servidos }\end{array}$ & $\mathrm{F}$ & $\begin{array}{l}18 \\
13\end{array}$ & EUA $^{12}$ \\
\hline $\begin{array}{l}{ }^{* *} \text { Melancia } x \text { suco de melancia } \\
\text { Coco x leite coco e leite } x \text { queijo }\end{array}$ & $\begin{array}{l}\text { Maior saciação e maior tendência à saciedade após alimentos } \\
\text { sólidos }\end{array}$ & $\begin{array}{l}\mathrm{F} \\
\mathrm{M}\end{array}$ & $\begin{array}{l}60 \\
60\end{array}$ & $\mathrm{EUA}^{48}$ \\
\hline $\begin{array}{l}\text { Almoço com pré-carga x almoço } \\
\text { sem pré-carga }\end{array}$ & $\begin{array}{l}\text { Redução de } 20 \text { \% na ingestão do almoço com a pré-cargas/ } \\
\text { Diferença no sabor e valor energético das pré-cargas }\end{array}$ & $\begin{array}{l}\mathrm{F} \\
\mathrm{M}\end{array}$ & $\begin{array}{l}30 \\
30\end{array}$ & $\mathrm{EUA}^{44}$ \\
\hline $\begin{array}{l}\text { Achocolatado líquido x semi-líqui- } \\
\text { do } \text { x semi-sólido }\end{array}$ & $\begin{array}{l}\text { \# Diferença na ingestão: líquidos > semi-líquidos > semi-sólidos/ } \\
\text { Menor tempo de ingestão do alimento líquido } \\
\text { \#\# Maior ingestão com líquido do que com semi-sólido/Ingestão } \\
\text { artificial (bomba de impulsão) tempo controlado }\end{array}$ & $\begin{array}{c}F \\
M \\
F \\
M\end{array}$ & $\begin{array}{c}72 \\
36 \\
35 \\
141\end{array}$ & Holanda ${ }^{20}$ \\
\hline Barra energética $\times$ shake & $\begin{array}{l}\text { Maior redução da fome após ingestão dos sólidos/variação de } \\
\text { macronutrientes e fibras e estudo com idosos }\end{array}$ & $\begin{array}{l}\mathrm{F} \\
\mathrm{M}\end{array}$ & $\begin{array}{l}7 \\
2\end{array}$ & EUA $^{43}$ \\
\hline
\end{tabular}

* Principais elementos não controlados com efeito potencial nos resultados, além das limitações de cada delineamento e características da amostra (participantes); n: número de indivíduos que participaram do estudo; sexo ( $F=f$ eminino e $M=$ masculino), idade etc. ${ }^{* *}$ Preparações utilizadas como pré-cargas $\rightarrow$ podem resultar em maior sensação de plenitude gástrica.

\# | Estudo (fora do laboratório); \#\# || Estudo (em laboratório). 
como o uso de clipes nasais pelos participantes para ingestão das dietas, pode ter também afetado esses resultados, além de as amostras ainda diferirem quanto à densidade energética e ao peso. Mais tarde, DiMeglio \& Mattes $^{49}$ contrastaram sólidos e líquidos na forma de refrigerante e doce. Uma compensação energética de cerca de 118\% foi verificada após o uso do doce, e nenhuma redução após o uso do refrigerante. Entretanto, não houve um controle do tempo de ingestão dos alimentos testados ao longo do dia. Posteriomente, Mattes \& Rothacker ${ }^{46}$ verificaram que a viscosidade de bebidas está inversamente relacionada à fome, corroborando os resultados encontrados recentemente por outro grupo de pesquisadores ${ }^{20}$. Porém, como foi salientado anteriormente, o delineamento desses estudos foi direcionado para uma comparação entre líquidos mais ou menos viscosos, e não entre sólidos e líquidos. Em 2007, outro estudo ${ }^{48}$ contrastou três grupos de alimentos sólidos e líquidos, tendo verificado que os alimentos líquidos testados, de forma geral, promoveram menor saciação e uma tendência à menor saciedade e que, especialmente em indivíduos obesos, isso ocorreu de forma mais pronunciada após a refeição hiperprotéica líquida. O Quadro 1 mostra, resumidamente, os principais estudos comparativos entre estado físico de alimentos e seus efeitos na ingestão alimentar.

\section{CONSIDERAÇÕES FINAIS}

Mediante a análise critica da literatura citada sobre este assunto, pode-se verificar que o estado físico do alimento tem grande influência nos níveis de fome e saciedade, e que existem fortes evidências de que os alimentos líquidos têm um fraco controle sobre o apetite, quando comparados aos sólidos. Vários trabalhos que observaram o efeito oposto apresentaram delineamento experimental inadequado, ou utilizaram sopas, iogurte, ou shake como veículos, não sendo exemplos adequados de alimentos na forma líquida.

Apesar de os mecanismos envolvidos nessa questão ainda não terem sido suficientemente caracterizados, acredita-se que fatores comportamentais, cognitivos, sensoriais, osmóticos, endócrinos, entre outros, estejam fortemente presentes e atuantes no prolongamento ou não da saciedade. Particularmente na obesidade, a interação desses fatores tem fundamental importância. Nesse sentido, o estado físico dos alimentos deve ser considerado com cautela, uma vez que os alimentos líquidos não promovem saciedade da mesma forma e intensidade que os sólidos, e parecem atuar também de forma diferenciada em indivíduos obesos e não obesos.

Portanto, em termos da prevenção e tratamento da obesidade, é necessário rever a freqüência e a quantidade do consumo de alimentos líquidos, contendo alto valor energético, especialmente na forma de bebidas.

\section{A GRADECIMENTOS}

Ao Conselho Nacional de Desenvolvimento Científico e Tecnológico e à Coordenação de Aperfeiçoamento de Pessoal de Nível Superior, pelo apoio financeiro concedido na forma de bolsas.

\section{COLABORADORES}

D.M. MOURÃO participou integralmente da elaboração e da redação do manuscrito, sendo o assunto abordado o principal foco de sua tese de doutorado. J. BRESSAN participou integralmente da elaboração do trabalho, como supervisora e orientadora, contribuindo substancialmente com a discussão e a apresentação do mesmo.

\section{REFERÊ NCIAS}

1. Mattes RD. Beverages and positive energy balance: the menace is the medium. Int J Obes (London). 2006; 30(Suppl 3):S60-5.

2. Mattes R. Fluid calories and energy balance: the good, the bad, and the uncertain. Physiol Behav. 2006; 89(1):66-70.

3. World Health Organization. Diet, nutrition and the prevention of chronic diseases. World Health Organ Tech Rep Ser. 2003; 916(1-8):1-149. 
4. Himaya A, Louis-Sylvestre J. The effect of soup on satiation. Appetite. 1998; 30(2):199-210.

5. Rolls BJ, Bell EA, Thorwart ML. Water incorporated into a food but not served with a food decreases energy intake in lean women. Am J Clin Nutr. 1999; 70(4):448-55.

6. Santangelo A, Peracchi M, Conte D, Fraquelli M, Porrini M. Physical state of meal affects gastric emptying, cholecystokinin release and satiety. $\mathrm{Br} J$ Nutr. 1998; 80(6):521-7.

7. Carmo MB, Toral N, Silva MV, Slater B. Consumo de doces, refrigerantes e bebidas com adição de açúcar entre adolescentes da rede pública de ensino de Piracicaba, São Paulo. Rev Bras Epidemiol. 2006; 9(1):121-30.

8. Popkin BM, Armstrong LE, Bray GM, Caballero B, Frei B, Willett WC. A new proposed guidance system for beverage consumption in the United States. Am J Clin Nutr. 2006; 83(3):529-42.

9. Vereecken CA, Inchley J, Subramanian SV, Hublet $A$, Maes $L$. The relative influence of individual and contextual socio-economic status on consumption of fruit and soft drinks among adolescents in Europe. Eur J Public Health. 2005; 15(3):224-32.

10. Storey ML, Forshee RA, Anderson PA. Beverage consumption in the US population. J Am Diet Assoc. 2006; 106(12):1992-2000.

11. Shields DH, Corrales KM, Metallinos-Katsaras E. Gourmet coffee beverage consumption among college women. J Am Diet Assoc. 2004; 104(4): 650-3.

12. Mattes R. Soup and satiety. Physiol Behav. 2005; 83(5):739-47.

13. Almiron-Roig E, Chen Y, Drewnowski A. Liquid calories and the failure of satiety: how good is the evidence? Obes Rev. 2003; 4(4):201-12.

14. Marciani L, Gowland PA, Spiller RC, Manoj P, Moore $R J$, Young $P$, et al. Gastric response to increased meal viscosity assessed by echo-planar magnetic resonance imaging in humans. J Nutr. 2000; 130(1):122-7.

15. Rumpler WV, Kramer M, Rhodes DG, Paul DR. The impact of the covert manipulation of macronutrient intake on energy intake and the variability in daily food intake in nonobese men. Int J Obes (Lond). 2006; 30(5):774-81.

16. Norton GN, Anderson AS, Hetherington MM. Volume and variety: relative effects on food intake. Physiol Behav. 2006; 87(4):714-22.

17. Ledikwe JH, Blanck HM, Kettel Khan L, Serdula MK, Seymour JD, Tohill BC, et al. Dietary energy density is associated with energy intake and weight status in US adults. Am J Clin Nutr. 2006; 83(6):1362-8.
18. Devitt AA, Mattes RD. Effects of food unit size and energy density on intake in humans. Appetite. 2004; 42(2):213-20.

19. Drewnowski A, Bellisle F. Liquid calories, sugar, and body weight. Am J Clin Nutr. 2007; 85(3):651-61.

20.Zijlstra N, Mars M, de Wijk RA, Westerterp-Plantenga MS, De Graaf C. The effect of viscosity on ad libitum food intake. Int J Obes (Lond). 2008; 32(4):676-83.

21. Fujise $T$, Yoshimatsu $H$, Kurokawa $M$, Oohara $A$, Kang $M$, Nakata $M$, et al. Satiation and masticatory function modulated by brain histamine in rats. Proc Soc Exp Biol Med. 1998; 217(2):228-34.

22. Sakata T, Yoshimatsu H, Masaki T, Tsuda K. Anti-obesity actions of mastication driven by histamine neurons in rats. Exp Biol Med (Maywood). 2003; 228(10):1106-10.

23. Yeomans MR, Symes T. Individual differences in the use of pleasantness and palatability ratings. Appetite. 1999; 32(3):383-94.

24. Yeomans MR, Gray RW, Mitchell CJ, True S. Independent effects of palatability and within-meal pauses on intake and appetite ratings in human volunteers. Appetite. 1997; 29(1):61-76.

25. Sawaya AL, Fuss PJ, Dallal GE, Tsay R, McCrory MA, Young $\mathrm{V}$, et al. Meal palatability, substrate oxidation and blood glucose in young and older men. Physiol Behav. 2001; 72(1-2):5-12.

26. LeBlanc J, Brondel L. Role of palatability on meal-induced thermogenesis in human subjects. Am J Physiol. 1985; 248(3 Pt 1):E333-6.

27. Crapo PA, Henry RR. Postprandial metabolic responses to the influence of food form. Am J Clin Nutr. 1988; 48(3):560-4.

28. Habas ME, MacDonald IA. Metabolic and cardiovascular responses to liquid and solid test meals. Br J Nutr. 1998; 79(3):241-7.

29. Mattes RD. Fluid energy-Where's the problem? J Am Diet Assoc. 2006; 106(12):1956-61.

30. Vincent $R$, Roberts $A$, Frier $M$, Perkins $A C$, MacDonald IA, Spiller RC. Effect of bran particle size on gastric emptying and small bowel transit in humans: a scintigraphic study. Gut. 1995; 37(2): 216-9.

31. Bergmann JF, Chassany O, Petit A, Triki R, Caulin C, Segrestaa JM. Correlation between echographic gastric emptying and appetite: influence of psyllium. Gut. 1992; 33(8):1042-3.

32. Moran TH. Cholecystokinin and satiety: current perspectives. Nutrition. 2000; 16(10):858-65.

33. Young JB, MacDonald IA. Sympathoadrenal activity in human obesity: heterogeneity of findings since 
1980. Int J Obes Relat Metab Disord. 1992; 16(12): 959-67.

34. Astrup A, Buemann B, Christensen NJ, Toubro S. Failure to increase lipid oxidation in response to increasing dietary fat content in formerly obese women. Am J Physiol. 1994; 266(4 Pt 1):E592-9.

35. Raben A, Astrup A. Leptin is influenced both by predisposition to obesity and diet composition. Int J Obes Relat Metab Disord. 2000; 24(4):450-9.

36. Cooling J, Barth J, Blundell J. The high-fat phenotype: is leptin involved in the adaptive response to a high fat (high energy) diet? Int J Obes Relat Metab Disord. 1998; 22(11):1132-5.

37. Wang G, Lee HM, Englander E, Greeley Jr. GH. Ghrelin-not just another stomach hormone. Regul Pept. 2002; 105(2):75-81.

38. Tschop M, Smiley DL, Heiman ML. Ghrelin induces adiposity in rodents. Nature. 2000; 407(6806): 908-13.

39. Masuda Y, Tanaka T, Inomata N, Ohnuma N, Tanaka $S$, Itoh $Z$, et al. Ghrelin stimulates gastric acid secretion and motility in rats. Biochem Biophys Res Commun. 2000; 276(3):905-8.

40. Druce MR, Wren AM, Park AJ, Milton JE, Patterson $M$, Frost $G$, et al. Ghrelin increases food intake in obese as well as lean subjects. Int J Obes (London). 2005; 29(9):1130-6.

41. Cummings DE, Purnell JQ, Frayo RS, Schmidova K, Wisse BE, Weigle DS. A preprandial rise in plasma ghrelin levels suggests a role in meal initiation in humans. Diabetes. 2001; 50(8):1714-9.

42. Tschop M, Weyer C, Tataranni PA, Devanarayan V, Ravussin E, Heiman ML. Circulating ghrelin levels are decreased in human obesity. Diabetes. 2001; 50(4):707-9.

43. Tieken SM, Leidy HJ, Stull AJ, Mattes RD, Schuster RA, Campbell WW. Effects of solid versus liquid meal-replacement products of similar energy content on hunger, satiety, and appetite-regulating hormones in older adults. Horm Metab Res. 2007; 39(5):389-94

44. Flood JE, Rolls BJ. Soup preloads in a variety of forms reduce meal energy intake. Appetite. 2007; 49(3):626-34

45. De Graaf C, Hulshof T. Effects of weight and energy content of preloads on subsequent appetite and food intake. Appetite. 1996; 26(2):139-51.

46. Mattes RD, Rothacker D. Beverage viscosity is inversely related to postprandial hunger in humans. Physiol Behav. 200; 74(4-5):551-7.

47.Zijlstra N, Mars M, De Wijk RA, Westerterp- plantenga MS, De Graaf C. Liquid foods result in higher ad libitum food intake than semi-solid foods because of a higher eating rate. Appetite. 2008; 50(2-3):567.

48. Mourao DM, Bressan J, Campbell WW, Mattes RD. Effects of food form on appetite and energy intake in lean and obese young adults. Int J Obes (London). 2007; 31(11):1688-95.

49. DiMeglio DP, Mattes RD. Liquid versus solid carbohydrate: effects on food intake and body weight. Int J Obes Relat Metab Disord. 2000; 24(6): 794-800

50. Hulshof T, De Graaf C, Weststrate JA. The effects of preloads varying in physical state and fat content on satiety and energy intake. Appetite. 1993; 21(3): 273-86

51. Havel PJ. Peripheral signals conveying metabolic information to the brain: short-term and long-term regulation of food intake and energy homeostasis. Exp Biol Med. 2001; 226(11):963-77.

Recebido em: 13/4/2007

Versão final reapresentada em: 31/12/2008 Aprovado em: 9/3/2009 